\title{
Myasthenia Gravis in Pregnancy: Case Series and Systematic Review
}

\author{
Harrison Banner ${ }^{1}$, Kirsten Niles ${ }^{1}$, Michelle Ryu ${ }^{2}$, Mathew Sermer ${ }^{1}$, Vera Bril ${ }^{3}$, and Kellie \\ Murphy ${ }^{1}$ \\ ${ }^{1}$ Sinai Health System \\ ${ }^{2}$ Mount Sinai Hospital Sidney Liswood Health Sciences Library \\ ${ }^{3}$ University of Toronto
}

August 23, 2020

\begin{abstract}
Background: Myasthenia gravis (MG) is an autoimmune disease which can affect reproductive-aged women and impact pregnancy outcomes. Objectives: To systematically review pregnancy outcomes for patients with MG. Search Strategy: Ovid MEDLINE, EMBASE, Cochrane Central Register of Controlled Trials, PubMed (Non-Medline records), Web of Science, and LILACS databases were systematically searched for pregnancies complicated by MG. Selection Criteria: Human studies of five or more subjects reporting outcomes of MG in pregnancy published in any language. Data Collection and Analysis: Following identification and review of relevant studies, data on study characteristics, MG subtypes, treatment, disease and pregnancy outcomes were extracted. Assessment of bias was performed using the National Institutes of Health Quality Assessment Tool for Case Series. In addition, cases of MG in pregnancy managed at our centre were identified and outcomes included in the analysis. Main Results: In total, 32 publications met inclusion criteria for systematic review, for a total of 33 unique data sets including 48 cases at our institution. In total, outcome data was available for 824 pregnancies complicated by MG. Overall risk of MG exacerbation was $33.8 \%$ with a $6.4 \%$ risk of myasthenic crisis in pregnancy and $8.2 \%$ postpartum. Spontaneous vaginal delivery occurred in $56.3 \%$ of pregnancies. The risk of transient neonatal myasthenia gravis (TNMG) was $13.0 \%$. Conclusions: One third of pregnant MG patients will experience an exacerbation with $6.4 \%$ and $8.2 \%$ experiencing myasthenic crisis in pregnancy and postpartum respectively. More than half of MG patients had a spontaneous vaginal birth. The risk of TNMG is $13 \%$
\end{abstract}

\section{Introduction:}

Myasthenia Gravis (MG) is an autoimmune disorder resulting from the binding of autoantibodies to acetylcholine receptors (AChR) at the neuromuscular junction. Patients experience fluctuating weakness in the skeletal muscles, primarily affecting the ocular, bulbar, limb, and respiratory muscles. Though uncommon, with a reported prevalence of 0.3 to 2.8 per $100,000^{1}$, MG incidence spikes for women during the $2^{\text {nd }}$ and $3^{\text {rd }}$ decades of life and therefore can impact pregnancy. Recent guidelines make recommendations about the optimal management of $\mathrm{MG}$ in pregnancy $^{2,3}$ though high-quality data is lacking.

MG typically has a relapsing and remitting course, with occasional exacerbations requiring more intensive treatment. Myasthenic crisis is a life-threatening complication involving weakness of the respiratory muscles leading to respiratory compromise requiring ventilatory support ${ }^{4}$.

MG treatment primarily involves symptomatic management with anticholinesterase agents, with steroids and other immunosuppressive agents used in more severe disease. For refractory cases or myasthenic crises, intravenous immunoglobulin (IVIG) and/or plasmapheresis can be employed. Thymectomy has been demonstrated to improve symptom control for patients with a thymoma and/or generalized seropositive disease ${ }^{5}$. 
MG has a variable course in pregnancy. Previous studies report exacerbation rates on the order of $30 \%{ }^{6,7}$ though higher ${ }^{8,9}$ and lower ${ }^{10}$ rates have been reported. The literature is similarly discordant in regards to pregnancy outcome, with some studies reporting increased rates of preterm prelabour rupture of membranes (PPROM), preterm birth (PTB), small for gestational age (SGA) babies and caesarean delivery ${ }^{11,12}$, and others reporting rates similar to that of the general population ${ }^{13,14}$. Transient neonatal myasthenia gravis (TNMG) is a complication whereby the AChR autoantibodies pass through the placenta causing a self-limited myasthenic syndrome in the neonate. Risk of TNMG is generally quoted to be 10-15\%, with individual series demonstrating rates ranging between $3-4 \%^{12,15}$ and $33 \%^{16}$.

The present study sought to systematically review all reported case series ([?]5 cases) of MG during pregnancy and to include a large case series from our institution in an effort to summarize and better understand the risks associated with MG in pregnancy.

\section{Methods:}

\section{Search Strategy}

The following electronic bibliographic databases were searched using a comprehensive search strategy developed by an information specialist (M.R.): (1) Ovid MEDLINE, (2) Ovid EMBASE, (3) Ovid EBM Reviews Cochrane Central Register of Controlled Trials, (4) PubMed (Non-Medline records), (5) Web of Science, and (6) LILACS (Latin American \& Caribbean Health Science Literature). Additionally, all registered controlled trials were screened in ClinicalTrials.gov, the WHO International Clinical Trials Registry Platform (ICTRP) and the International Standard Randomised Controlled Trial Number Registry (ISRCTNR). The search strategy was structured according to the 2015 Peer Reviewed Electronic Search Strategies (PRESS) Guidelines. Subject headings and free text terms related to 'myasthenia gravis', 'pregnancy' and 'obstetric delivery' were included. No restrictions were applied to publication year, language, or age. All databases were searched for relevant references published up to July 31, 2020. All references and duplicate records were managed and screened using EndNote citation management software and Covidence systematic review software (Covidence systematic review software, Veritas Health Innovation, Melbourne, Australia. www.covidence.org).

The review was registered in the PROSPERO database (2019; registration number CRD42019143072) prior to study initiation. The methods were performed in accordance with the Preferred Reporting Items for Systematic Reviews and Meta-Analyses (PRISMA) guidelines (http://www.prisma-statement.org).

Inclusion criteria included randomized controlled trials, prospective or retrospective cohort studies and case series of at least five subjects with MG in pregnancy. Exclusion criteria included reviews without individual patient-level data, publication of data reported in previous publications, and abstract presentations.

\section{Data Extraction and Assessment of Risk of Bias}

Two authors (HB and KMN) independently screened all titles and abstracts. Relevant studies were then pulled for full text review. These two authors independently reviewed, extracted data and assessed risk of bias, using "Quality Assessment Tool for Case Series" (NIH.gov), for each full text article. Disagreements were settled via consensus or if necessary, with discussion involving a third author (KEM).

Data was extracted from included studies using a standardized data extraction form. Extracted data included disease information, including MG treatment, proportion of pregnancies with exacerbation, stable disease, and remission in pregnancy, and the rate of myasthenic crisis during pregnancy and up to six weeks postpartum. Data was also extracted regarding pregnancy outcomes, including mode of delivery as well as the rate of pregnancy complications, including: preterm birth $<34$ weeks and $<37$ weeks gestational age, preterm prelabour rupture of membranes, small for gestational age $<10 \%$ ile (based on birthweight for given gestational age), and the proportion of neonates experiencing transient neonatal myasthenia gravis.

\section{Institutional Case Series}

After local Research Ethics Board approval (MSH REB \#19-0183-C, July 26, 2019), a retrospective chart review of all pregnant women with myasthenia gravis cared for at Mount Sinai Hospital, Toronto, was 
conducted from the Medical Disorders of Pregnancy clinic database from January $1^{\text {st }} 2000$ to August $31^{\text {st }} 2019$.

Cases were included if there was a documented diagnosis of myasthenia gravis made prior to or during the pregnancy based on clinical or electrophysiological criteria. Cases of congenital myasthenic syndromes were excluded as this was felt to represent a separate disease entity. Only cases which were followed for the duration of pregnancy at our institution with available birth outcomes were included.

For the purposes of this study, each pregnancy was treated as a separate case for patients having more than one pregnancy. Although multiple pregnancies in the same individual patient will have correlated outcomes, the previously published literature on this topic included subsequent pregnancies in the same patient and it was not possible to assign pregnancies to individuals in these studies. Therefore, this convention was followed in our case series to maintain consistency. This was felt to be reasonable as comparative statistics were not planned.

For each case, data regarding demographics, diagnosis, MG treatment, disease course during pregnancy and up to six weeks postpartum, mode of delivery, and maternal-fetal-neonatal outcomes were collected. Disease course was defined as "remission" if the patient had an improvement in their symptoms or remained asymptomatic throughout the pregnancy, "unchanged" if symptoms were stable throughout pregnancy, and "exacerbation" if the patient experienced a documented increase in the frequency or severity of symptoms or required an increase in medication dose or an additional treatment modality in pregnancy. Neonates were considered to be small for gestational age if their birthweight was less than the $10^{\text {th }}$ percentile for gestational age based on the growth curves created by Kramer et $\mathrm{al}^{17}$.

\section{Statistical Methods}

Descriptive statistics were performed using SPSS (IBM SPSS Statistics, Version 23.0, Armonk, NY: IBM Corp).

\section{Results:}

Our literature search yielded 980 citations. After removing 334 overlapping results, 646 titles and abstracts were screened for inclusion. Of these, 227 were retrieved for full-text review and 32 ultimately were included in our review (Fig 1). Our case series data was included in the final review for a total of 33 unique data sets involving MG in pregnancy.

In our institutional case series, 39 patients with MG had 48 pregnancies. Table 1 summarizes the demographic information, MG treatment, and clinical course for each pregnancy at our centre.

The data collected from the 32 studies $^{8}, 13,18-47$ included in the systematic review as well as our own case series are summarized in Table 2. The included studies represent a heterogeneous group from 22 different countries with case data collected from 1947 up until 2020. Twenty-eight of the included studies were case series, four used a retrospective cohort design, and one case-control study was included. Sample sizes ranged from 5 to 163 subjects with MG in pregnancy. There was significant variability in the type of data collected between studies. The significant heterogeneity in study design and reporting precluded meaningful comparative analysis of the study data.

Risk of bias was assessed and this is summarized in Figure 2. Due to the nature of methodology used for these studies, the overall risk of bias is quite high.

\section{MG Treatment in Pregnancy}

The proportion of patients with a thymectomy prior to pregnancy ranged from $16.7 \% 36$ to $100 \% 37,39$. Across studies, the majority of patients were treated with anti-cholinesterase medications during pregnancy, though a significant number also required prednisone $34,40,47$. More recent studies were more likely to include patients treated with Azathioprine ${ }^{40}, \mathrm{IVIG}^{8}$, or plasmapheresis ${ }^{42}$.

\section{MG Course in Pregnancy:}


Across all studies reporting this outcome, 181/535 (33.8\%) patients experienced an exacerbation of their MG during pregnancy, while 242/505 (47.9\%) had stable disease and 100/505 (19.8\%) had an improvement in their symptoms from baseline.

In total, $23 / 357$ (6.4\%) patients experienced an episode of myasthenic crisis in pregnancy, and 31/380 (8.2\%) experienced myasthenic crisis during the postpartum period.

\section{Mode of Delivery:}

The overall rate of C/S done for MG was 25/824 (3.0\%), with an additional 249/824 (30.2\%) patients having a $\mathrm{C} / \mathrm{S}$ for an obstetrical indication. The rate of operative vaginal delivery was $86 / 824$ (10.4\%), with 464/824 $(56.3 \%)$ of patients having a spontaneous vaginal birth.

\section{Pregnancy Complications:}

Preterm birth less than 34 and 37 weeks' gestation occurred in 14/327 (4.3\%) and 69/579 (11.9\%) pregnancies respectively. The overall risk of PPROM was 27/404 (6.7\%).

In studies providing information on birthweight for gestational age, 55/390 (14.1\%) neonates were smaller than the $10^{\text {th }}$ percentile. The risk of transient neonatal myasthenia gravis was $89 / 686(13.0 \%)$.

\section{Discussion:}

\section{Main Findings:}

Myasthenia gravis is an uncommon neuromuscular condition which can have a significant impact on pregnancy. Previous publications have described a wide range of risk of MG exacerbation during pregnancy, ranging in individual samples from $0 \%^{37}$ to $60 \%^{41,48}$ depending on the study. In our systematic review, $33.8 \%$ of MG patients overall experienced an exacerbation of their symptoms during pregnancy, a finding consistent with the generally quoted risk of $30-45 \%^{6,7}$. Previous authors have stated that exacerbation is most likely to occur in the first trimester and in the postpartum period ${ }^{21}$, though the current review did not examine the timing of exacerbation within pregnancy since the timing of exacerbation was only reported in a small minority of studies.

Myasthenic crisis, an important and potentially life-threatening complication of MG, occurred in $6.4 \%$ of patients during pregnancy and in $8.2 \%$ of patients during the postpartum period.

The method by which MG patients give birth shows significant regional and temporal variation in the previously published literature, with the proportion of patients having a vaginal birth ranging from $0 \%^{36}$ to $100 \%^{35}$ in some small series. Overall, $56.3 \%$ of births across all included studies occurred by SVD and an additional $10.4 \%$ were operative vaginal deliveries. In total, $33.3 \%$ of deliveries were via $\mathrm{C} / \mathrm{S}$, with $30.2 \%$ done for an obstetrical indication. Only 3.0\% of patients had C/S with MG as the indication.

For patients with symptomatic MG at the time of birth, there is some concern that the voluntary striated muscles used during active pushing may be weakened by MG exacerbation and further compromised by excessive maternal effort, precipitating myasthenic $\operatorname{crisis}^{49}$. For patients with exacerbation symptoms at the time of delivery, an assisted second stage of labour may decrease the need for maternal effort and minimize this risk. This has not been universally adopted as standard practice, and indeed current guidelines note that spontaneous vaginal delivery is the objective and should be encouraged ${ }^{2}$.

Patients with MG seem to be at increased risk of PPROM compared to the $3 \%^{50}$ risk in the general population, with this complication occurring in $6.7 \%$ of all MG pregnancies. This finding has been previously reported $^{21,40}$, though a pathophysiologic explanation remains elusive.

Whether or not MG increases the risk of preterm labour and birth has been the subject of previous controversy in the literature, with some populations, our own included, appearing to demonstrate an elevated risk ${ }^{33}$. Our systematic review did not find an elevated risk of PTB in MG patients, with overall risks of PTB of $11.9 \%$ prior to 37 weeks and $4.3 \%$ prior to 34 weeks, which is similar to the population risk ${ }^{51}$. Tanacan et 
al have previously suggested that an elevated risk of PTB may exist in the population of MG patients who experience exacerbation in pregnancy ${ }^{21}$ though this was not assessed in the current study.

Previous studies have demonstrated conflicting findings regarding the association between MG and fetal growth, with high rates of small for gestational age babies reported in some series ${ }^{13},{ }^{26},{ }^{29}$ but not others $8,29,42$. Our review found that $14.1 \%$ of babies born to mothers with myasthenia gravis had an infant with a birthweight less than the $10^{\text {th }}$ percentile for gestational age overall.

Finally, the risk of transient neonatal myasthenia gravis in individual studies has ranged from $0 \%^{18,26,36,37,47}$ to $33.3 \%^{34,39,42}$. In our large sample, the overall rate was $89 / 686(13.0 \%)$. Previous studies have demonstrated a lack of correlation between anti-AChR titre ${ }^{43}$, duration of illness and treatment modalities $^{34}$ and the risk of TNMG. The current understanding of risk factors for this complication remains limited. Patients should be counselled about this risk and parents and care providers should be aware of the signs of this condition and the fact that there is no known association between this outcome and any predictive risk factor.

\section{Strengths and Limitations:}

The current systematic review represents an accumulation of data, which to our knowledge has not previously been synthesized systematically. We used a search strategy which encompassed multiple databases and did not eliminate studies based on publication language which allowed us to capture a diverse group of data sets. By excluding studies with sample sizes less than 5, some of the bias inherent in the publication of extreme case studies was minimized. This study extracted data on a large range of factors related to the treatment and outcomes of MG in pregnancy, and due to the large total sample represents the best estimate of the true risk presently available.

The heterogenous nature of the included studies means that data is represented from many different geographical regions. The fact that we did not use time or geographical exclusions increases the generalizability of our data, but does mean that our findings include regional and temporal variations in practice.

The main limitation of a study of this nature is the inclusion of low-quality evidence, as the majority of included studies were small and did not use a comparative design. Case series are particularly vulnerable to convenience sampling and selection bias, and may not appropriately represent the wider population of patients with MG embarking on pregnancy. The lack of a comparator group in the majority of included studies compromises internal validity and means that results are presented in a descriptive manner rather than employing meaningful comparative statistical analysis. Finally, most studies reported on pregnancies rather than by individual patients. It is possible that patients with less severe disease and fewer complications overly contributed to this review and therefore lead to an underestimation of the potential risks in pregnancy. Clinicians should use caution in implementing the findings of this study, though the inherent limitations are a function of the types of studies in the literature and are similar for most uncommon conditions in pregnancy.

\section{Interpretation:}

For patients with MG entering pregnancy, the current study allows the current best estimate for use in counselling regarding disease behaviour as well as the risk of myasthenic and pregnancy complications.

\section{Conclusion:}

Myasthenia gravis has important implications and considerations for pregnancy which should be discussed with patients. The overall risk of exacerbation in pregnancy is approximately $33 \%$, with a small but significant subset experiencing potentially life-threatening myasthenic crisis in pregnancy or postpartum. Patients with $\mathrm{MG}$ are at increased risk of requiring assisted vaginal delivery or $\mathrm{C} / \mathrm{S}$ compared to the general population. MG does not appear to significantly increase the rate of other pregnancy complications, with the exception of PPROM, which occurs at a rate roughly double that of the general population. Neonates should be observed for signs of TNMG, regardless of antenatal risk factors or maternal status.

\section{Disclosure of Interests:}


VB has previously received consultancy fees, honoraria and research support from UCB, Takeda, CSL, Grifols, Alexion, Argenx, Alnylam, Immunovant, Sanofi, Momenta and Biogen.

The other authors have no conflicts of interest to declare.

\section{Contribution to Authorship:}

HB and KEM conceived, designed, and oversaw the conduct of the study. HB wrote the initial draft of the manuscript, conducted abstract and full-text screening, conducted risk of bias assessment, and synthesized the data. KMN conducted screening of abstracts and full texts and conducted risk of bias assessment. MR developed and carried out the search strategies. MS, VB, and KEM provided clinical and methodological input. All authors critically revised the article and approved the final version for publication.

Funding: The work presented in this manuscript did not receive funding from any agency.

\section{References:}

1. Deenan JCW HC, Verschuuren JJGM, Verbeek ALM. The epidemiology of neuromuscular disorders: a comprehensive overview of the literature. Journal of neuromuscular diseases. 2015;2:73-85.

2. Sanders DB WG, Benatar M, Evoli A, Gilhus NE, Illa I, Kuntz N, Massey JM, Melms A, Murai H, Nicolle M, Palace J, Richman DP, Verschuuren J, Narayanaswami P. International consensus guidance for managment of myasthenia gravis. Neurology. 2016;87(4):419-25.

3. Sussman J FM, Maddison P, Hill M, Leite MI, Hilton-Jones D. The Association of British Neurologists' myasthenia gravis guidelines. Annals of the New York Academy of Sciences. 2018;1412(1):166-9.

4. Ahmed S KJ, Janjua N, Alkawi A, Khatri I, Yahia AM, Souyah N, Qureshi AI. An update on myasthenic crisis. Current Treatment Options Neurology. 2005;7(2):129-41.

5. Melzer N RT, Fuhr P, Gold R, Hohlfeld R, Marx A, Melms A, Tackenberg B, Schalke B, Schneider-Gold C, Zimprich F, Meuth SG, Wiendl H. Clinical features, pathogenesis and treatment of myasthenia gravis: a supplement to the Guidelines of the German Neurological Society. Journal Neurology. 2016;263(8):1473-94.

6. Djelmis J SM, Mayer D, Ivanisevic M. Myasathenia gravis in pregnancy: report on 69 cases. European Journal of Obstetrics and Gynecology and Reproductive Biology. 2002;104:21-5

7. Tellez-Zenteno JF H-RL, Salinas V, Estanol B, da Silva O. Myasathenia gravis and pregnancy: clinical implications and neonatal outcome. BMC Musculoskeletal Disorders. 2004;5(42).

8. Braga AC, Pinto C, Santos E, Braga J. Myasthenia gravis in pregnancy: Experience of a portuguese center. Muscle \& nerve. 2016;54(4):715-20.

9. Qi Q-W, Wang D, Liu J-T, Bian X-M. [Management of pregnancy with myasthenia gravis: 7 cases report]. Zhonghua fu chan ke za zhi. 2012;47(4):241-4.

10. Kostera-Pruszczyk A E-SB. Macierzynstwo chorych na miastenie. Gin Pol. 2005;76:122-5.

11. Ducci RD, Lorenzoni PJ, Kay CSK, Werneck LC, Scola RH. Clinical follow-up of pregnancy in myasthenia gravis patients. Neuromuscular disorders : NMD. 2017;27(4):352-7.

12. Hoff JM DA, Gilhus NE. Myasthenia gravis consequences for pregancy, delivery, and the newborn. Neurology. 2003;61:1362-6.

13. Wen JC, Liu TC, Chen YH, Chen SF, Lin HC, Tsai WC. No increased risk of adverse pregnancy outcomes for women with myasthenia gravis: a nationwide population-based study. European Journal of Neurology. 2009;16(8):889-94. 
14. Perucca E CE, Ricci P, Altamirano R, Cuadra C, Saez D, Carvajal R, Galleguillos I, Szigethi M, Alvarez S, Agurto N, Zamora J. Miastenia gravis: embarazo e impacto perinatal. Rev Chil Obstet Ginecol. 2006;71(3):201-6.

15. de Assis JL MP, Zambon AA, Scaff M Miastenia grave na gravidez e parto. Neurobiol Recife. $1983 ; 46(1): 3-12$.

16. Eden RD GS. Myasthenia gravis and pregnancy: A reappraisal of thymectomy. Obstetrics and Gynecology. 1983;62(3):328-33.

17. Kramer MS PR, Wen SW, Joseph KS, Allen A, Abrahamowicz M, Blondel B, Breart G, for the Fetal/Infant Health Study Group of the Canadian Perinatal Survellance System. A new and improved population-based canadian reference for birth weight for gestational age. Pediatrics. 2001;108(2):e35-e42.

18. Tsurane K, Tanabe S, Miyasaka N, Matsuda M, Takahara M, Ida T, et al. Management of labor and delivery in myasthenia gravis: A new protocol. The journal of obstetrics and gynaecology research. 2019;45(5):974-80.

19. Thoulon JM, Galopin G, Seffert P, Garin JP, Dumont M. [Myasthenia and pregnancy (author's transl)]. Myasthenie et grossesse. 1978;7(8):1395-403.

20. Tellez-Zenteno JF, Hernandez-Ronquillo L, Salinas V, Estanol B, da Silva O. Myasthenia gravis and pregnancy: clinical implications and neonatal outcome. BMC musculoskeletal disorders. 2004;5:42.

21. Tanacan A, Fadiloglu E, Ozten G, Gunes AC, Orgul G, Beksac MS. Myasthenia gravis and pregnancy: retrospective evaluation of 27 pregnancies in a tertiary center and comparison with previous studies. Ir J Med Sci. 2019;188(4):1261-7.

22. Slama A, Tourni A, Tarmiz K, Dhafer BL, Khaled BJ. Regional anaesthesia for labour and delivery in the myasthenic patient: Report of five cases. Annales Francaises D Anesthesie Et De Reanimation. 2008;27(2):180-1.

23. Shi BB, Zeng LC. Management of myasthenia gravis during pregnancy: A report of eight cases. Open Life Sciences. 2018;13(1):28-33.

24. Roth TC, Raths J, Carboni G, Rosler K, Schmid RA. Effect of pregnancy and birth on the course of myasthenia gravis before or after transsternal radical thymectomy. European journal of cardio-thoracic surgery : official journal of the European Association for Cardio-thoracic Surgery. 2006;29(2):231-5.

25. Ramirez C, de Seze J, Delrieu O, Stojkovic T, Delalande S, Fourrier F, et al. [Myasthenia gravis and pregnancy: clinical course and management of delivery and the postpartum phase]. Myasthenie auto-immune et grossesse: evolution clinique, accouchement et post-partum. 2006;162(3):330-8.

26. Qi QW, Wang D, Liu JT, Bian XM. [Management of pregnancy with myasthenia gravis: 7 cases report]. Zhonghua fu chan ke za zhi. 2012;47(4):241-4.

27. Podciechowski L, Brocka-Nitecka U, Dabrowska K, Bielak A, Hadacz B, Wilczynski J. Pregnancy complicated by Myasthenia gravis - twelve years experience. Neuro endocrinology letters. 2005;26(5):603-8.

28. Picone O, Audibert F, Gajdos P, Fernandez H. [Myasthenia gravis and pregnancy. Report on 13 cases]. Myasthenie et grossesse: a propos de 13 grossesses. 2003;32(7):654-9.

29. Perucca P E, Cuellar J E, Ricci A P, Altamirano A R, Cuadra C C, Sáez M D, et al. Miastenia gravis: embarazo e impacto perinatal. Rev chil obstet ginecol. 2006;71(3):201-6.

30. Mitchell PJ, Bebbington M. Myasthenia gravis in pregnancy. Obstetrics and gynecology. 1992;80(2):17881.

31. Miller HJ, Sellmann AH, Mickal A. Myasthenia gravis of pregnancy at charity hospital. J La Med Soc. 1968;120(5):231-4. 
32. Kostera-Pruszczyk A, Emeryk-Szajewska B. [Myasthenia gravis and pregnancy]. Macierzynstwo chorych na miastenie. 2005;76(2):122-6.

33. Hoff JM, Daltveit AK, Gilhus NE. Myasthenia gravis: Consequences for pregnancy, delivery, and the newborn. Neurology. 2003;61(10):1362-6.

34. Gveric-Ahmetasevic S, Colic A, Elvedji-Gasparovic V, Gveric T, Vukelic V. Can neonatal myasthenia gravis be predicted? Journal of perinatal medicine. 2008;36(6):503-6.

35. Gordon SV, Peven PS. Myasthenia gravis and pregnancy. Grace Hospital Bulletin. 1968;46(2):44-50.

36. Garcia SA, Ogata AJ, Patriota RG, Lippi UG, Grabert HH. Myasthenia gravis and pregnancy: review of the literature and report of 6 cases occurring in 5 patients. Revista paulista de medicina. 1989;107(3):144-8.

37. Gamez J, Salvado M, Casellas M, Manrique S, Castillo F. Intravenous immunoglobulin as monotherapy for myasthenia gravis during pregnancy. Journal of the neurological sciences. 2017;383:118-22.

38. Fraser D, Turner JWA. Myasthenia gravis and pregnancy. Lancet. 1953;2(9):417-9.

39. Eden RD, Gall SA. Myasthenia gravis and pregnancy: a reappraisal of thymectomy. Obstetrics and gynecology. 1983;62(3):328-33.

40. Ducci RD, Lorenzoni PJ, Kay CSK, Werneck LC, Scola RH. Clinical follow-up of the pregnancy in myasthenia gravis patients. Journal of Neuromuscular Diseases. 2016;3(Supplement 1):S194.

41. Drazancic A, Jusic A, Jurenic D. [Myasthenia gravis and pregnancy]. Myasthenia gravis i trudnoca. 1978;26(1-4):41-6.

42. Djelmis J, Sostarko M, Mayer D, Ivanisevic M. Myasthenia gravis in pregnancy: report on 69 cases. European journal of obstetrics, gynecology, and reproductive biology. 2002;104(1):21-5.

43. Cheng I, Lin C-H, Lin M-I, Lee J-S, Chiu H-C, Mu S-C. Outcome of myasthenia gravis mothers and their infants. Acta paediatrica Taiwanica = Taiwan er ke yi xue hui za zhi. 2007;48(3):141-5.

44. Chabert L, Benhamou D. Myasthenia gravis, pregnancy and delivery: A series of ten cases. Annales Francaises d'Anesthesie et de Reanimation. 2004;23(5):459-64.

45. Castro RFe, Cunha SPd, Duarte G, Nogueira AA, Mauad Filho F, Castro PSAFe. Miastenia gravis e gravidez: análise de 9 casos. Rev bras ginecol obstet. 1995;17(1):34-42.

46. Assis JL, Marchiori PE, Zambon AA, Scaffl M. Miastenia grave na gravidez e parto. Neurobiologia. $1983 ; 46(1): 3-12$.

47. Almeida C, Coutinho E, Moreira D, Santos E, Aguiar J. Myasthenia gravis and pregnancy: anaesthetic management-a series of cases. European journal of anaesthesiology. 2010;27(11):985-90.

48. Chabert L, Benhamou D. [Myasthenia gravis, pregnancy and delivery: a series of ten cases]. Myasthenie, grossesse et accouchement: a propos de dix cas. 2004;23(5):459-64.

49. Uncu G KI, Ozan H, Cengiz C. Pregnancy and myasthenia gravis. A case report. Clin Exp Obstet Gynecol. 1995;22(2):145-7.

50. Smith G RC, Anand N, Brennan B, Connors G, Crane J, Fraser W, Gratton R, Moutquin JM, Scott H, Schneider C, Walker M. Prevalence, management, and outcomes of preterm prelabour rupture of membranes of women in Canada. Journal of Obstetrics and Gynaecology Canada. 2005;27(6):547-53.

51. Blencowe H CS, Oestergaard MZ, Chou D, Moller AB, Narwal R, Adler A, Garcia CV, Rohde S, Say L, Lawn JE. National, regional, and worldwide estimates of preterm birth rates in the year 2010 with time trends since 1990 for selected countries: a systematic analysis and implications. Lancet. 2012;379(9832):2162-72.

\section{Hosted file}


2020_08_17_MG_Paper_BJOG_Fig 1_PRISMA.pptx available at https://authorea.com/users/352615/ articles/476782-myasthenia-gravis-in-pregnancy-case-series-and-systematic-review

\section{Hosted file}

2020_08_19_MG_Paper_Table1_CS.xlsx available at https://authorea.com/users/352615/articles/ 476782-myasthenia-gravis-in-pregnancy-case-series-and-systematic-review

\section{Hosted file}

2020_08_04_MG_paper_BJOG_Table2_SR.xlsx available at https://authorea.com/users/352615/ articles/476782-myasthenia-gravis-in-pregnancy-case-series-and-systematic-review

\section{Hosted file}

MG_Paper_BJOG_Fig 2_Risk of Bias_July18.pptx available at https://authorea.com/users/352615/ articles/476782-myasthenia-gravis-in-pregnancy-case-series-and-systematic-review 